\title{
Plástica da valva aórtica em pacientes portadores de insuficiência aórtica: resultados imediatos e tardios
}

\author{
Marcondes T. NEVES JÚNIOR*, Pablo M. A. POMERANTZEFF*, Carlos M. A. BRANDÃO*, \\ Max GRINBERG*, Miguel BARBERO-MARCIAL*, Noedir A. G. STOLF*, Geraldo VERGINELLI*, \\ Adib D. JATENE*
}

RBCCV $44205-304$

\begin{abstract}
Neves Júnior M T, PomerantzeffPM A, Brandăo C M A, Grinberg M, Barbero-Marcial M, Stolf N A G, Verginelli G. Jatene A D - Plástica da valva aórtica em pacientes portadores de insuficiência aórtica: resultados imediatos e tardios. Rev Bras Cir Cardiovasc 1996; 11 (3): 155-60.

RESUMO: No período de agosto de 1980 a maio de 1995 , foram estudados 31 pacientes que apresentavam insuficiência aórtica e que foram submetidos à plástica da valva aórtica (P V A). Dezoito $(58,06 \%)$ pacientes eram do sexo masculino e $13(42,94 \%)$ do sexo feminino. A média de idades foi de 20.9 $+/-18,3$, coma idade variando de 2 a 68 anos. A etiologia das lesర̋es foi congênita em $21(67,65 \%)$ pacientes, reumática em $6(19,35 \%)$, degenerativa em $3(9,67 \%)$ e endocardite infecciosa em $1(3,25 \%)$. O tipo de plástica realizada foi: plicatura junto às comissuras com fixaçăo na parte externa da aorta em 10 pacientes, plicatura junto às comissuras com fixaçăo interna das vávulas em 10, anuloplastia parcial em 4, plicatura valvular central em 5 e correção valvular com placa de pericárdio bovino em 2. As principais operaçōes associadas foram: ventriculosseptoplastia em 14 e plástica de valva mitral em 7 pacientes. O tempo médio de pinçamento aórtico foi de 70,53 minutos. O tempo médio de circulação extracorpórea (CEC) foi de 96,43 minutos. Não houve mortalidade operatória. Um paciente foi submetido a nova plàstica no mesmo ato operatório para a correçăo de insuficiência aórtica residual. Um paciente faleceu após 19 meses de insuficiência cardiaca. Vinte e cinco $(80,6 \%$ ) apresentam-se em classe funcional I (NYH A) no pós-operatório tardio. Podemos concluir que os pacientes submetidos à $\mathrm{P} V \mathrm{~A}$ apresentaram baixo risco e boa evoluçâo tardia.
\end{abstract}

DESCRITORES: Insuficiência da valva aórtica, cirurgia. Valva aórtica, cirurgia. Valva aórtica, plástica.

\section{INTRODUÇĀO}

A cirurgia conservadora na insuficiência aórtica ainda apresenta um desenvolvimento técnico inferior em relacão à cirurgia das valvas atrioventriculares, Isto se justifica pelas dificuldades inerentes às características anatômicas da valva aórtica (1).

Com a observação das limitaçōes existentes com o implante de próteses, sejam biológicas ou metálicas, além das complicaçőes relacionadas ao uso de anticoagulantes, principalmente em pacientes jovens, está havendo uma mudança de postura frente a pacientes com insuficiência aórtica, objetivando, principalmente, a durabilidade da correção cirúrgica. Poucos Serviços apresentam uma casuística expressiva de cirurgia conservadora da valva aórtica em pacientes adultos portadores de insuficiência aórtica; em contrapartida, há um número expressivo de trabalhos sobre este tipo de procedimento em crianças com defeito do septo ventricular (2).

O presente trabalho tem como objetivo demonstrar a experiência do Instituto do Coração do Hospital das Clínicas da Faculdade de Medicina da Universidade de São Paulo (InCór) com a plástica de

Trabalho realizado no Instituto do Coraçăo do Hospital das Clínicas da Faculdade de Medicina da Universidade de São Paulo, São Paulo, SP, Brasil. Apresentado ao $23^{\circ}$ Congresso Nacional de Cirurgia Cardiaca. Recife, 20 a 23 de março, 1996.

- Do Instituto do Coraço do Hospital das Clínicas da Faculdade de Medicina da Universidade de São Paulo.

Endereço para corrospondencia: Marcondes Tavares Neves Jünior. Av. Enéas de Carvalho Aguiar, 44. Cerqueira Cesar. Săo Paulo, SP, Brasil. CEP: 05403-000. 
Neves Júnior M T, Pomerantzeff PM A, Brandão C M A, Grinberg M, Barbero-Marcial M, Stolf N A G, Verginelli G, Jatene A D-PIástica da valva aórtica em pacientes portadores de insuficiência aórtica: resultados imediatos e tardios. Rev Bras Cir Cardiovasc $1996 ; 11(3): 155-60$.

valva aórtica, em pacientes portadores de insuficiência aórtica.

\section{CASUISTICA E MÉTODOS}

Foram estudados 31 pacientes submetidos à plástica da valva aórtica, no período de agosto de 1980 a maio de 1995 no InCór, sendo 18 (58,06\%) do sexo masculino e $13(41,94 \%)$ do sexo feminino. A média de idades dos pacientes foi de 20,9 e 0 desvio padräo de $+/-18,3$ anos, tendo variado de 2 a 68 anos.

O diagnóstico pré-operatório foi de insuficiência aórtica em todos os pacientes. O diagnóstico etiológico foi de origem congênita em $21(67,75 \%)$ pacientes, febre reumática em $6(19,35 \%)$, degeneraçäo em $3(9,67 \%)$ endocardite infecciosa em 1 $(3,25 \%)$. O principal diagnóstico associado foi a comunicaçāo interventricular (CIV) subaórtica em 12 pacientes; outros diagnósticos associados foram insuficiência coronária em 1 , dupla disfunçāo mitral em 1 , estenose mitral em 3 , insuficiência mitral em 3 , insuficiência tricúspide em 2, aneurisma do seio de Valsalva em 2, fístula aorta-ventrículo esquerdo em 1, CIV subpulmonar em 1, CIV trabecular perimembranosa em 1, estenose subaórtica em 4, estenose infundibular de VE em 1, estenose pulmonar em 2, persistência de canal arterial em 2, tronco arterioso em 1, valva aórtica bivalvulada em 2 e presença de prótese mitral em 2 pacientes.

Os principais métodos diagnósticos utilizados foram: o ecocardiograma bidimensional quantificado pelo Doppler nos 31 pacientes no pré e no pósoperatório, o cateterismo cardíaco em 22 pacientes no pré-operatório e o ecocardiograma transesofágico no intra-operatório em 10 pacientes.

No pré-operatório 3 pacientes se encontravam em classe funcional I (N Y H A), 12 em classe funcional 11,10 em classe funcional III e 6 em classe funcional IV.

O tipo de plástica realizada foi: plicatura junto às comissuras com fixaçāo na parte externa da aorta (3) em 10 pacientes, plicatura junto às comissuras com fixação na parte interna das válvulas em 10 (Figura 1), tendo um dos pacientes valva aórtica bivalvulada, anuloplastia parcial em 4 (Figura 2), correção com placa de pericárdio bovino em 2, plicatura valvular em 5 , que foi associada a ressecçāo valvular em 2 pacientes e a plicatura junto às comissuras com fixaçăo na parte interna das vávulas em 1 paciente o qual também possuía valva bivalvulada (Figura 3 ).

Os principais procedimentos cirúrgicos associados à PVA encontram-se na Tabela 1 , sendo os principais a ventriculosseptoplastia em 14 pacientes

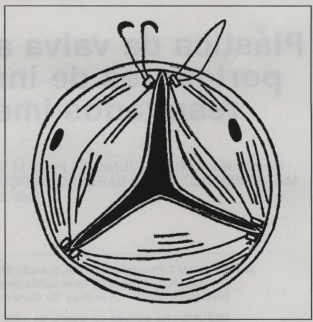

Fig. 1 - Plicatura junto as comissuras com fixação na parte interna das válvulas.

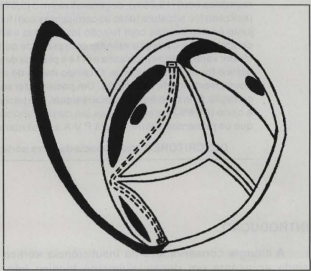

Fig. 2 - Anuloplastia parcial.

e a plástica de valva mitral em 7 pacientes.

Todos os pacientes foram operados com auxilio da circulaçăo extracorpórea. O tipo de cardioplegia utilizada foi a cristaloide filtrada. O tempo médio de pinçamento aórtico foi de 70,53 minutos e o tempo médio de CEC foi de 96,43 minutos.

\section{RESULTADOS}

As principais válvulas envolvidas, de acordo com a etiologia, foram a coronariana direita no grupo de 
Neves Júnior M T, Pomerantzeff PM A, Brandão C M A, Grinberg M, Barbero-Marcial M, Stolf N A G, Verginelli G, Jatene A D-Plástica da valva aórtica em pacientes portadores de insuficiência aórtica: resultados imediatos e tardios. Rev Bras Cir Cardiovasc $1996 ; 11(3): 155-60$.

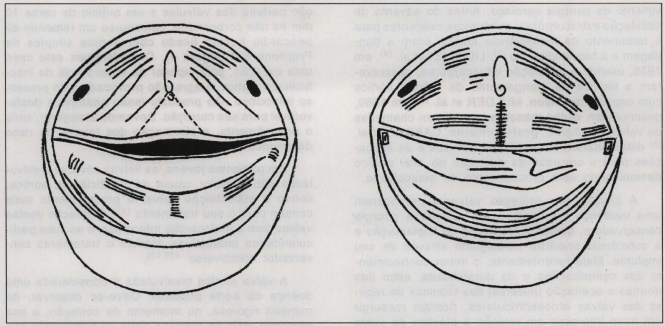

Fig. 3 - Ressecção válvular associada a plicatura junto às comissuras com fixação na parte interna das válvulas.

etiologia congênita, com 14 pacientes e a não coronariana em 6 no grupo de etiologia reumática; os outros dois grupos, devido ao número reduzido de pacientes, não foram analizados.

O tempo médio de seguimento pós-operatório destes pacientes foi de 30,54 meses, com o desvio padrão de $+/-24,7$, sendo o período mínimo de 5 meses e o máximo de 84 meses.

Não houve mortalidade operatória. Um paciente submetido a plicatura junto às comissuras com fixaçāo na face interna das válvulas apresentou rotura dos pontos da comissura, diagnosticada no intraoperatório através do ecocardiograma transesofágico, sendo corrigida imediatamente.

As complicaçōes pós-operatórias foram: derrame pleural em 2 pacientes, derrame pericárdico em

TABELA 1

PROCEDIMENTOS CIRUURGICOS ASSOCIADOS

\begin{tabular}{lrr}
\hline CIRURGIAS ASSOCIADAS & $N^{9}$ & $\%$ \\
\hline Ventriculosseptoplastia & 14 & 37,83 \\
Correçăo de Estenose Subaórtica & 4 & 10,82 \\
Ligadura de Canal Arterial & 2 & 5,41 \\
Remoça de Estenose Infundibular de VE & 1 & 2,70 \\
Ressecçāo de Banda Anómala de VD & 1 & 2,70 \\
Troca de Tubo de Pericárdio Bovino n-18 & 1 & 2,70 \\
Plástica de Valva Mitral & 7 & 18,91 \\
Retroca de Valva Mitral & 2 & 5,41 \\
Plástica de De Vega em Valva Tricúspide & 2 & 5,41 \\
Correção de Aneurisma do Seio de Valsalva & 2 & 5,41 \\
Correçăo de Fistula Ao-VE & 1 & 2,70 \\
\hline
\end{tabular}

1. paralisia frênica em 1 e pneumonia em 1 . Um paciente foi submetido a reoperação, devido a sangramento localizado na aorta.

Todos os pacientes foram submetidos ao ecocardiograma bidimensional quantificado pelo Doppler no período de pós-operatório durante a sua internaçäo, sendo estes comparados com os realizados no pré-operatório; foram considerados três graus de insuficiência aórtica: leve, moderada e grave. Todos os pacientes apresentaram redução no grau de insuficiência aórtica no período de pós-operatório.

Em relação à classe funcional ( $N$ Y H A), nenhum paciente deixou de apresentar melhora no pós-operatório, encontrando-se 3 pacientes assintomáticos, 22 pacientes em classe funcional $1,5 \mathrm{em}$ classe funcional II e 1 em classe funcional III.

No seguimento dos pacientes houve apenas um óbito, 19 meses após a operação, devido a insuficiência cardiaca congestiva, em paciente com etiologia reumática, e submetido a plástica da valva aórtica com plicatura da válvula não coronariana além de retroca de prótese mitral e plástica da valva tricúspide; apresentava no pré-operatório classe funcional IV (N Y H A) e, ao ecocardiograma, insuficiência aórtica moderada.

\section{COMENTÁRIOS}

A cirurgia conservadora da valva aórtica teve sua evoluçăo diretamente relacionada ao desenvol- 
Neves Júnior M T, Pomerantzeff PM A, Brandāo C M A, Grinberg M, Barbero-Marcial M, Stolf NA G, Verginelli G, Jatene A D-Plástica da valva aórtica em pacientes portadores de insuficiência aórtica: resultados imediatos e tardios. Rev Bras Cir Cardiovasc 1996; 11 (3): $155-60$.

vimento da cirurgia cardíaca. Antes do advento da circulação extracorpórea, as técnicas existentes para - tratamento da insuficiência aórtica eram a bandagem $\theta$ a bicuspidizaçăo (1). LILLEHEI et al. (4), em 1958 , usando a circulação extracorpórea, descreveram a técnica de alongamento da valvula aórtica com esponja de Ivalon. MULDER et al, (5), em 1960. descreveram várias manobras que foram chamadas de valvuloplastias e, posteriormente, CABROL et al. (6) determinaram os principios técnicos e as indicaçōes para a correçāo da dilataçāo do anel aórtico denominando este procedimento de anuloplastia.

A criaçāo das próteses valvares provocaram uma mudança de conduta em relação à cirurgia conservadora, devido à facilidade de implantação e à suficiência imediata conseguida através de seu implante. Mais recentemente, o maior conhecimento das complicaçōes e da durabilidade, além das normas e aceitaçāo universal das técnicas de reparo das valvas atrioventriculares, fizeram ressurgir um novo interesse em relação à plástica da valva aórtica (1).

Os fatores limitantes para que a plástica de valva aórtica seja considerada como primeira opção no tratamento cirúrgico de sua insuficiència săo: o seu mecanismo, que em alguns casos não está bem definido, a existência de menor quantidade de tecido valvar sadio que permita uma variedade de técnicas para sua reconstrução e também as dificuldades técnicas para a avaliaçăo do resultado final da plástica no intra-operatório, pois a visão direta através da aortotomia não assegura o sucesso do procedimento (2).

As técnicas utilizadas para a correção da insuficiência aórtica se dividem em dois grupos: as plásticas, onde as válvulas săo submetidas a manobras combinadas com o objetivo de suspensăo ou correçāo de sua redundância, e o seu alongamento, que é realizado geralmente com material sintético, de preferência o pericárdio bovino. Esta última técnica tem indicação em valvas com insuficiência importante, de pequena espessura, com retraçőes grosseiras que ocupam mais da metade da área valvular $(1.7-9)$

As contra-indicaçōes para o uso destas técnicas sāo a infecção ativa, a calcificaçāo valvular, a anatomia desfavorável que dificulte a reconstruçăo (10), e as valvas bivalvuladas, que săo, ainda, motivo de controvérsia.

$\mathrm{Na}$ vigência de endocardite ativa, poderemos considerar a possibilidade da plástica somente em lesōes localizadas, geralmente representadas por perfuraçōes. Em nossa casuística, tivemos 1 paciente submetido a este tratamento após um episódio de endocardite. Apresentava uma valva com coapta- ção perfeita das válvulas e um orifício de cerca 10 mm na năo coronariana; colocou-se um remendo de pericárdio bovino fixado com pontos simples de Propilene 6-0 (Figura 4). Consideramos este caso uma exceção, pois, apesar da importância da insuficiência aórtica, a agressão provocada pelo processo infeccioso năo produziu lesão anatômica desfavorável para sua correção. Devemos assegurar, após o procedimento, a eliminação dos fatores de risco da endocardite.

Em pacientes jovens, as valvas aórticas bivalvuladas săo a maior causa de insuficiência aórtica, sendo a substituição valvar o procedimento mais comum para o seu tratamento (11). A relaçäo destas valvas com a endocardite infecciosa, e as suas particularidades anatômicas, tornam o tratamento conservador controverso $(12,13)$.

A valva aórtica bivalvulada é considerada uma doença da aorta proximal. Deve-se observar, de maneira rigorosa, no momento da correçăo, a sua anatomia, pois os espaços entre as comissuras são diferentes, assim como o tamanho das válvulas e de suas bordas livres; a base das válvulas fundidas se insere um pouco acima da base da válvula regular, devendo-se observar, ainda, o tamanho do anel valvar e dos seios de Valsalva (11). Estas particularidades anatômicas explicam porque, após a plástica durante a análise estática, a valva possa parecer suficiente $e$, a seguir, quando o coração retorna à sua condiçäo dinâmica, apresente um grau de insuficiência variável. MOIDL et al. (11), estudando, através do ecocardiograma transesofágico, 14 pacientes que possuiam valvas aórticas bivalvuladas, que foram submetidos à plástica, encontraram 2 pacientes que apresentaram insuficiência importante no

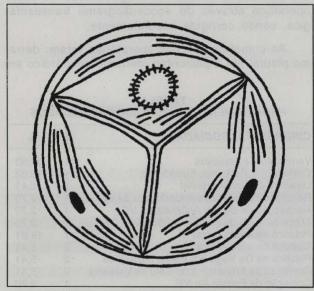

Fig. 4 - Plástica com remendo de pericárdio bovino. 
Neves Júnior M T, PomerantzeffPM A, Brandão C M A, Grinberg M, Barbero-Marcial M, Stolf NA G, Verginelli G, Jatene A D - Plástica da valva aórtica em pacientes portadores de insuficiência aórtica: resultados imediatos e tardios. Rev Bras Cir Cardiovasc $1996 ; 11(3): 155-60$.

pós-operatório, sendo que em 1 não foi encontrada a causa desta insuficiência. Nesta casuística, 3 pacientes apresentaram um aumento do grau de insuficiência aórtica após 1 ano $\mathrm{e}$, em todos, foi observado aumento do tamanho da junção sinotubular, sendo, em 1, de $11 \mathrm{~mm}$. Em nossa casuística, apenas 2 pacientes possuíam valva aórtica bivalvulada, tendo evoluido no pós-operatório com insuficiência aórtica discreta, avaliada através do ecocardiograma e em classe funcional I (N Y H A). Năo existem relatos na literatura em relaçāo à durabilidade a longo prazo da PVA em pacientes com valva aórtica bivalvulada, mas o estudo da história natural de 41 pacientes com esta característica demonstrou que, em 11 anos, $61 \%$ permaneciam estáveis.

Nos pacientes com etiologia reumática, as leső̉es mais freqüentes eram devido à redundância ou ao desabamento das válvulas não coronariana e coronariana esquerda. Este grupo apresentava classe funcional ( $\mathrm{N}$ Y H A) mais elevada e lesōes mitrais, sendo que 2 pacientes já possuiam próteses biológicas nesta posiçāo, e todos sofreram intervençōes sobre esta valva no momento da PVA. Este foi o único grupo de pacientes que apresentou mortalidade, com 1 óbito 19 meses após a operaçăo, devido a insuficiência cardiaca congestiva. A evoluçăo tardia destes pacientes mostrou que a PVA não se deteriora como nas lesōes mitrais, pois a insuficiência aórtica residual permaneceu tal como no pós-operatório imediato, quando analizada pelo ecocardiograma Doppler. AMANO (14), analizando um grupo de pacientes submetido a PVA $\theta$ outro submetido a plástica da valva mitral, observaram evolução semelhante nos dois grupos, em relaçāo à classe funcional, no pós-operatório, à mortalidade e às complicaçōes, considerando semelhante a durabilidade das plásticas nos dois grupos. DURAN et al. (1), estudando pacientes com etiologia reumática, demonstraram uma durabilidade de 11 anos.

Os pacientes com lesöes degenerativas apresentavam um perfil pré-operatório semelhante ao do grupo reumático, sendo submetidos, em todos os casos, à plástica da valva mitral associada à plástica da valva aórtica. A evolução pós-operatória destes pacientes foi diferente do grupo reumático, em relação à regressăo da classe funcional, pois todos os pacientes voltaram à classe funcional I ( $N$ Y H A), enquanto os pacientes do outro grupo apresentaram classe funcional mais elevada.

No grupo com etiologia congênita, o principal diagnóstico foi a CIV subaórtica em 12 pacientes, sendo a ventriculosseptoplastia a operação associa- da mais freqüente. A média de idades dos pacientes submetidos a ventriculosseptoplastia associada a PVA foi de 13, 2 anos. As principais técnicas cirúrgicas utilizadas foram a de TRUSLER et al. ${ }^{(3)}$ e a plicatura junto às comissuras com fixaçăo na parte interna das válvulas. A evolução pós-operatória destes pacientes foi satisfatória, geralmente apresentando um grau discreto de insuficiência aórtica, com melhora em relação à classe funcional. OKITA et al. (15) consideram contra-indicado a realizaçäo de PVA em pacientes acima de 15 anos de idade portadores de insuficiência aórtica associada a CIV, pois reconhecem que o defeito septal ao longo dos anos provoca dilatação anular, retração e, algumas vezes, perfuração valvular que provocam, após a operaçăo, uma evolução diferente da apresentada pelos pacientes mais jovens, necessitando de troca valvar em menor tempo. Em nossa casuistica, foram realizadas 5 plásticas em pacientes com idade acima de 15 anos, com evoluçäo da insuficiência aórtica de discreta para moderada ao ecocardiograma em 3 pacientes, num período de cerca de 32 meses, sem comprometimento da classe funcional. A experiência neste grupo mostrou que a plástica tem boa durabilidade se as lesōes valvares nāo forem avançadas no momento da operação.

Com o reconhecimento da ineficácia da avaliação do grau de insuficiência valvar através da aortotomia logo após o término da plástica, alguns autores estăo desenvolvendo um método alternativo de visão direta através de um valvoscópio, ainda sob pinçamento aórtico, com a aorta parcialmente aberta. Este método parece ser promissor, mas carece de relatos na literatura, além da vantagem da análise da valva em sua posição original, sem necessitar do fechamento total da aorta permitir modificação na plástica, sem aumento significativo do tempo cirúrgico (1). Os autores $(1,2)$ que estāo desenvolvendo o valvoscópio advogam o uso do ecocardiograma transesofágico no intra-operatório para a análise do resultado da plástica, onde săo observados os mecanismos de regurgitação, sua origem, a direção do jato regurgitante e a movimentação das válvulas, tendo como única desvantagem a necessidade de fechamento total da aortotomia e a retirada da pinça da aorta.

Podemos concluir que a plástica da valva aórtica é operação segura, com baixo índice de morbidade e mortalidade; o respeito às suas indicaçōes e limitações leva ao sucesso, com boa evoluçăo tardia, propiciando um dos principais objetivos da cirurgia valvar que é a manutenção da valva original, já que a correção das alteraçōes anatômicas e funcionais a torna hemodinamicamente semelhante à valva normal. 
Neves Jủnior M T, Pomerantzeff PM A, Brandāo C M A, Grinberg M, Barbero-Marcial M, Stolf NA G, Verginelli G, Jatene A D - Plástica da valva aórtica em pacientes portadores de insuficiência aórtica: resultados imediatos e tardios. Rev Bras Cir Cardiovasc $1996 ; 11(3) ; 155-60$

RBCCV $44205-304$

Neves Júnior M T, Pomerantzeff PM A, Brandāo C MA, Grinberg M, Barbero-Marcial M, StolfN A G, Verginelli G. Jatene A D - Valvuloplasty for aortic insulficiency: immediate and late results. Rev Bras Cir Cardiovasc 1996:11 (3): 155-60.

ABSTRACT: Between August 1980 and May 1995 we studied 31 patients who underwent aortic valvuloplasty for aortic insutficiency. Eighteen $(58.06 \%)$ were male and $13(42.94 \%)$ female. The age ranged from 2 to 68 years (avarage $20.9+/-18.3$ ). The ethiology was congenital in $21(67.65 \%$ ), rheumatic in 6 $(19.35 \%)$, degenerative in $3(9.67 \%)$ and infective endocarditis in $1(3.25 \%)$. The type of valvuloplasty performed were: anuloplasty with external fixation of the cusp in 10, anuloplasty with internal fixation of the cusp in 10. partial anuloplasty in 4, triangular resection of prolapsed cusp in 5 and correction with a patch of bovine pericardium in 2 patients. The most frequent associated surgery was ventriculosseptoplasty in 14. The mean time of cardiopulmonary bypass and aortic crossclamp was 96.43 and $70.53 \mathrm{~min}$, respectively. We didn't have operatory death. One patient was submitted to a new valvuloplasty in the intra-operative period because of a residual insufficiency. We observed one death in the follow-up due to a cardiac insufficiency nineteen months after surgery. Twenty five $(80.6 \%)$ patients are in functional class (NYH A) in the follow-up. We concluded that patients who underwent aortic valvuloplasty for aortic insufficiency had low surgical risk and good outcome.

DESCRIPTORS: Aortic valve insufficiency, surgery. Aortic valve, surgery. Aortic valvuloplasty.

\section{REFERÉNCIAS BIBLIOGRÁFICAS}

1 Duran C, Kumar N, Gometza B, Hobes Z A - Indications and limitations of aortic valve reconstruction. Ann Thorac Surg 1991; 52: 447-54.

2 Cosgrove M D, Rosenkranz E R, Hendren W G, Bartlett J C. Stewart W J - Valvuloplasty for aortic insufficiency. J Thorac Cardiovasc Surg 1991; 102 . $571-7$.

3 Trusler G A, Moes A F, Kidd B S - Repair of ventricular septal defect with aortic insufficiency. I Thorac Cardiovasc Surg 1973; 66: 394-403.

4 Lillehei C W, Gott V L, DeWall R A, Varco R L. The surgical treatment of stenotic or regurgitant lesion of the mitral and aortic valves by direct vision utilizing a pump oxygenator. I Thorac Cardiovasc Surg 1958; 35: 154-91.

5 Mulder D G, Kattus A A, Longmire W P. The treatment of acquired aortic stenosis by valvuloplasty. $J$ Thorac Cardiovasc Surg 1960; 40: 731-43.

6 Cabrol A, Guiraudon G, Bertrand M - Le traitement de I' insuffisance aortique par l'annuloplastie aortique. Arch Mal Coour 1966; 9: 1305-12.

7 Duran C M G - Reconstrurive techniques for rheumatic aortic valve disease. J Cardiac Surg 1988; 3: 23-8.

8 Duran C M G, Gometza B, Kumar N, Gallo R, Bjornstad K - From aortic cusp extension to valve replacement with Stentless pericardium. Ann Thorac Surg 1995: 60: $428-32$.

9 Yacoub M, Fajan A, Stassano P. Radley S R - Results of valve conserving operations for aortic regurgitation. Circulation 1993; 68 (Pt 2): II321.

10 Carpentier A - Cardiac valve surgery: the "French Correction". J Thorac Cardiovasc Surg 1983; 86 323-37.

11 Moidl R, Moritz A, Simon P, Kupilik N, Wolner E, Mohi $W$ - Ecocardiografic results after repair of incompetent bicuspid aortic valves. Ann Thorac Surg 1995; 60: 669-72.

12 Mills P, Leech G, Davies M, Leatham A - The Natural History of nonstenotic bicuspid aortic valve. Br Heart $J$ 1978; 40: 951-7

13 Roberts W C, Morrow A G, Mcintosh C L, Jones M, Epstein S E - Congenitally biscupid aortic valve causing severe, pure aortic regurgitation without superimposed infective endocarditis. Am J Cardiol 1981; 47: 206-9.

14 Amano J, Suzuki A, Sunamori M - Results of reconstructive surgery for acquired valve disease: evaluation of mitral and aortic valvuloplasty. Thorac Cardiovasc Surgeon 1994; 42: 9-13.

15 Okita $Y$, Miki S, Kusuhara $K$ et al, - Long term results of aortic valvuloplasty for aortic regurgitation associated with ventricular septal defect. JThorac Cardiovasc Surg 1988; 96: 769-74. 